\title{
Rare Recurrence of Traumatic Carotid Cavernous Fistula after Parent Artery Occlusion-Report of Two Cases and Review of Literature
}

\author{
Savith Kumar ${ }^{1}$ Virender Malik $\quad$ Jayadevan Enakshy Rajan ${ }^{1} \quad$ Santhosh Kumar Kannath
}

'Department of Imaging Sciences and Interventional Radiology, Neurointervention Center, Sree Chitra Tirunal Institute of Medical Sciences and Technology, Trivandrum, Kerala, India

\begin{abstract}
Address for correspondence Dr. Santhosh Kumar Kannath, MD, PDCC, Department of Imaging Sciences and Interventional Radiology, Neurointervention Center, Sree Chitra Tirunal Institute of Medical Sciences and Technology, Trivandrum 695011, Kerala, India (e-mail: drsanthoshkannath@gmail.com).
\end{abstract}

\begin{abstract}
The authors present two cases of recurrence of traumatic carotid cavernous fistula (CCF) following complete exclusion by parent artery occlusion (PAO). In both cases, the fistula recurred through the development of indirect CCF and reconstitution of the

Keywords

- carotid cavernous fistula

- parent artery occlusion

- posttraumatic

- recurrence

- vasa vasorum occluded artery through the development of vasa vasorum or simple recanalization of the parent artery. The cavernous venous sac was patent in both cases. The patent cavernous sac along with the inflammatory or angiogenetic factors might have induced dural neovascularization leading to the development of indirect CCF. These factors along with ischemia of the arterial wall secondary to the steal phenomenon due to persistent shunt flow would have triggered the development of vasa vasorum. Thus the cavernous sac embolization may have to be considered in addition to PAO when PAO is planned as a therapeutic option for direct CCF.
\end{abstract}

\section{Introduction}

Carotid cavernous fistula (CCF) is an abnormal direct or indirect communication between the intracranial part of internal carotid artery (ICA) and or external carotid artery (ECA) with the cavernous sinus (CS). Based on the etiology, the direct CCF is classified as traumatic or spontaneous. Endovascular treatment of direct CCF includes several options such as embolization using balloons or coils, sealing of the rent using covered stents or newly introduced flow diverters..$^{1,2}$ Endovascular procedure has a high success rate of 88 to $98 \%$ with no to low complication rate. ${ }^{3}$ Endovascular parent vessel occlusion (PAO) is an alternative option done in selected situations in which reconstruction of the artery is difficult. ${ }^{1}$ The recurrence rate in the treatment of traumatic CCF varies between 10 and $15 \%$, and this is primarily observed in cases treated with detachable balloon embolization. The failure may be attributed to premature balloon deflation, rupture, or migration. ${ }^{1,3}$ However, there are only very few instances of recurrence of CCF following endovascular PAO 4,5 We present two cases of CCF with recurrence following complete exclusion by PAO. The factors predisposing to this unusual occurrence are discussed.

\section{Case Report}

Case 1

A 55-year-old man was involved in a road traffic accident (RTA) and sustained a head injury, which required intensive case unit (ICU) admission for management. Computed tomography (CT) showed fractures involving the left temporal bone and left the orbital wall. The patient developed insidious onset redness and proptosis in the left eye 2 weeks after RTA, which was suspected to be CCF. Magnetic resonance imaging (MRI) showed the prominence of the left CS and bilateral superior ophthalmic veins (SOVs). Digital subtraction angiography (DSA) confirmed the presence of direct CCF in left cavernous ICA and a pseudoaneurysm of the left middle meningeal artery (MMA) ( - Fig. 1A, B). He underwent endovascular intervention with PAO for left direct CCF and coil embolization of left MMA pse udoaneurysm ( Fig. 1C, D). received

August 7, 2017

accepted after revision

October 25, 2017

published online

March 22, 2018
DOI https://doi.org/

10.1055/s-0037-1609056.

ISSN 2457-0214.
Copyright $\odot$ by Indian Society of

Vascular and Interventional

Radiology
License terms

()(1) $\Theta \circledast$ 


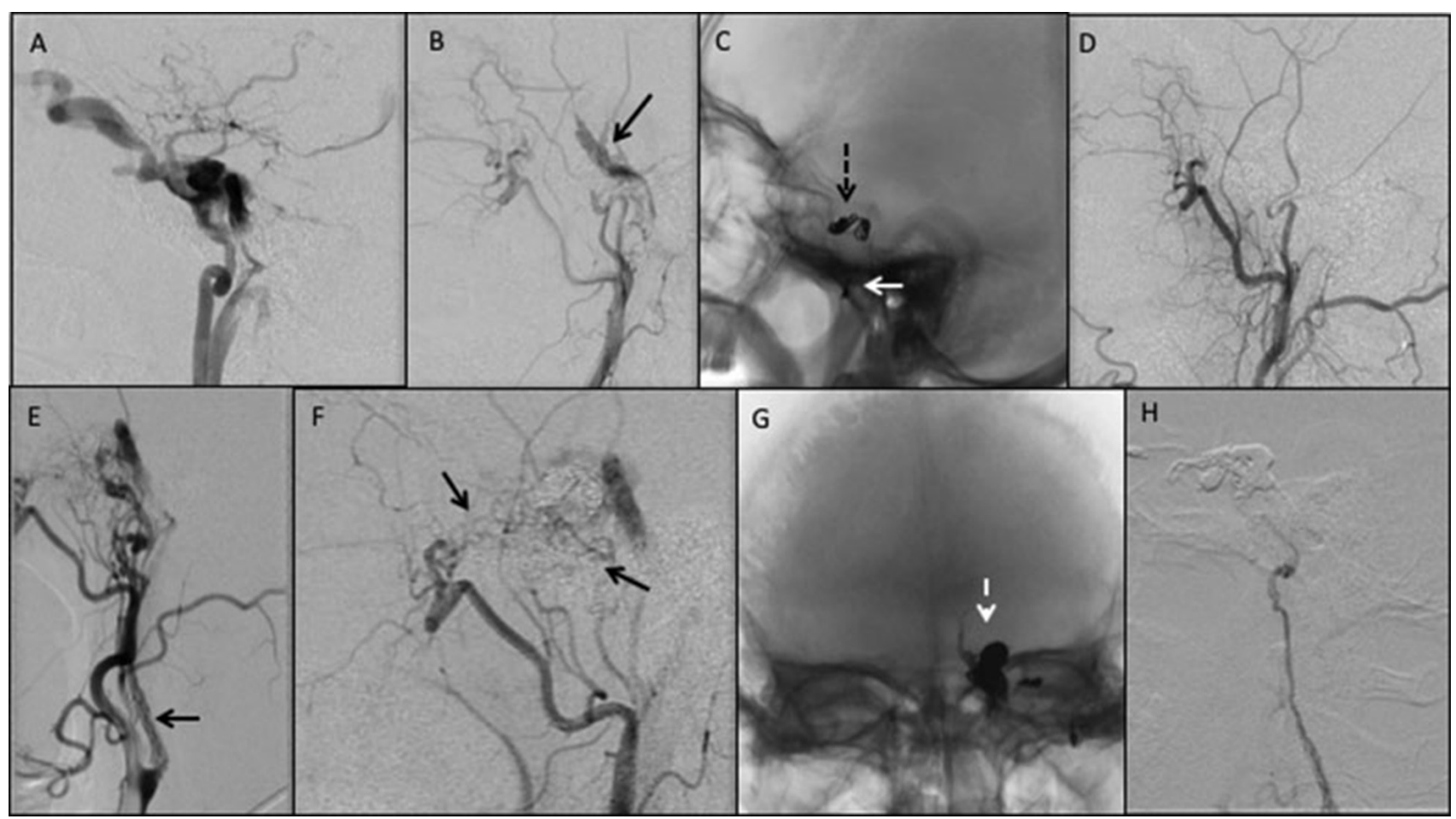

Fig. 1 Angiogram and intervention during the initial presentation (A-D). Left ICA angiogram (A) lateral view shows direct CCF with anterior, posterior, and cortical venous reflux. Left ECA angiogram (B) lateral view shows traumatic pseudoaneurysm of the MMA (black arrow). Radiograph of skull (C) AP view shows coil mass of PAO (dashed black arrow) and MMA pseudoaneurysm embolization in the left middle cranial fossa (white arrow). Left CCA angiogram postembolization (D) shows a complete cessation of the shunt and no opacification of the left ICA. Angiogram and intervention during the recurrence (E, F). Left CCA angiogram (E) lateral view shows recurrence of the left CCF with recanalization of the ICA via vaso vasorum (black arrow). Left ECA angiogram (F) shows distal IMA and MMA branches contributing to the fistula (black arrows). Radiograph of the skull (G) shows coil mass and Squid cast in the left parasellar region from transvenous embolization of the left cavernous sinus (dashed white arrow). Left CCA angiogram postembolization $(\mathbf{H})$ shows complete cessation of the shunt.

PAO was performed using detachable coils, and an immediate postintervention check angiogram revealed complete cessation of the shunt ( - Fig. 1C, D). He was asymptomatic for the next 5 months when his symptoms of left eye proptosis and redness recurred. Check DSA revealed recurrence of the CCF on the left side ( $\mathbf{- F i g}$. 1E, F). The arterial feeders were from distal branches of left internal maxillary (IMA), clival branches of left ascending pharyngeal arteries, and left MMA (-Fig. 1F). Minimal supply from distal branches of right IMA and meningohypophyseal branches of right ICA was also noted. The left ICA showed recanalization through vasa vasorum, which also contributed to the fistula (-Fig. 1E). Transvenous embolization of the left CS was performed with coils and Squid 18 liquid embolic device (Emboflu), achieving complete obliteration of the sac and cessation of the shunt (-Fig. 1G). Check angiogram done 5 days postintervention showed no evidence of residual fistula. The patient remained asymptomatic until the last clinical follow-up at 2 years.

\section{Case 2}

A 25-year-old man was involved in an RTA and sustained maxillofacial and head injuries. One day later, he developed pulsatile proptosis and chemosis in the right eye. CT scan showed prominent bilateral SOV and mandibular fracture, and DSA examination revealed a right direct CCF ( - Fig. 2A). He underwent detachable balloon embolization of the venous sac; however, because of the protrusion of the balloon through the rent into the parent artery and resultant flow compromise, PAO was considered to avoid thromboembolic complications. PAO was performed using detachable coils (-Fig. 2B-D). Postprocedure period, he had good symptomatic improvement with subsequent resolution of his proptosis and chemosis. On 17th postprocedure day, he developed recurrence of right eye proptosis and chemosis that gradually progressed. Follow-up DSA showed recurrence of his right CCF with supply anterogradely from the recanalized right ICA as well as from the terminal branches of bilateral IMA (-Fig. 2E, F). Transvenous coiling of the right CS was done resulting in complete cessation of the shunt ( - Fig. 2G, H).

\section{Discussion}

The mainstay of treatment of CCF is by endovascular approaches, and the choice of endovascular treatment is dependent on various factors such as the angioarchitecture of the fistula, the size of the rent, degree of the shunt, operator's preference, and institutional experience.

The recurrence rate following treatment of direct CCF has been very low, as shown by various studies. ${ }^{3}$ Most of the recurrence occurred with the use of the detachable balloons, caused by premature deflation or rupture and occurring within days of treatment. Recurrence after ICA occlusion/PAO has been seen due to reverse filling of thefistula through the distal ICA. ${ }^{5}$ Studies have shown recurrence 


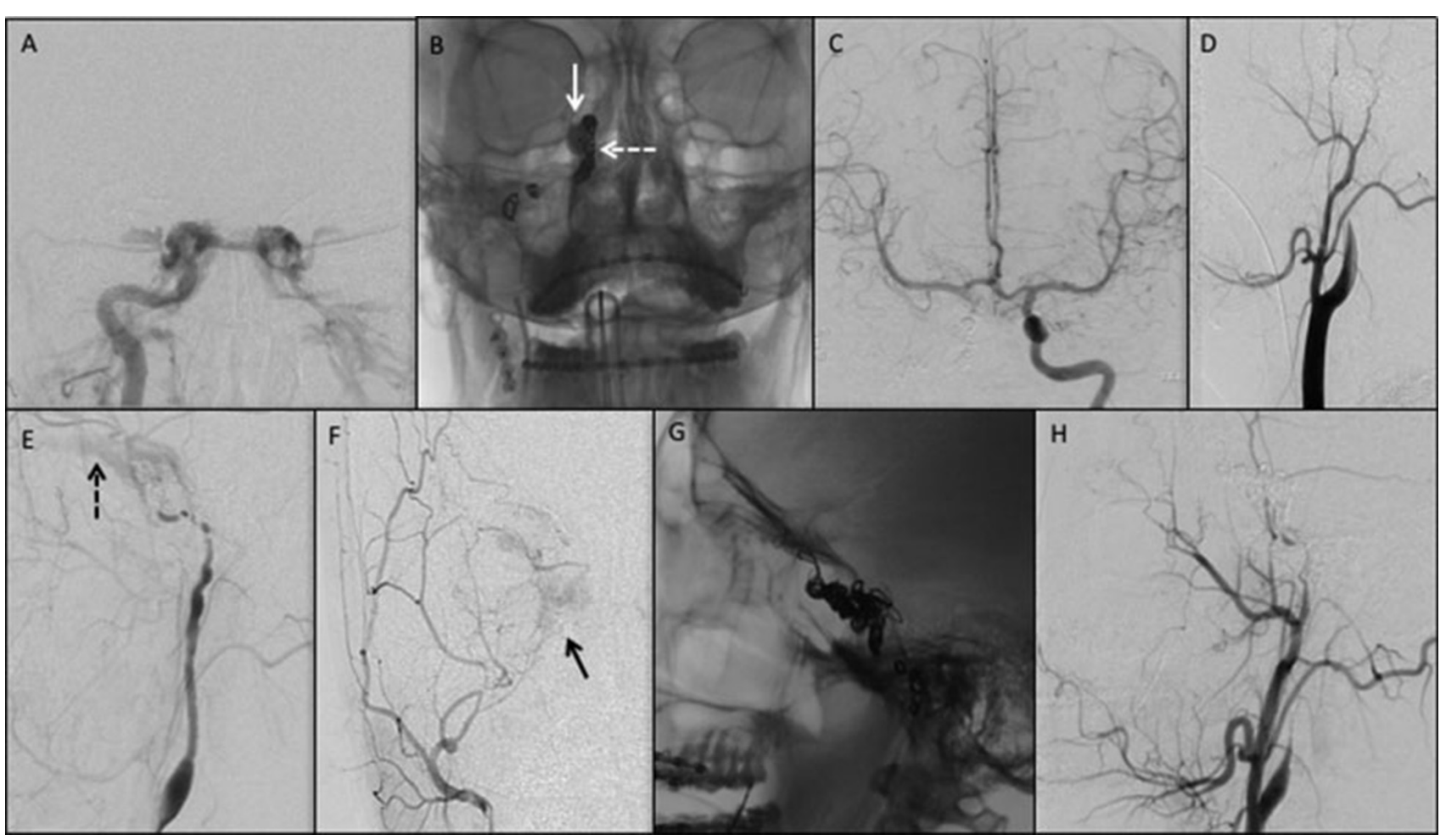

Fig. 2 Angiogram and intervention during initial presentation (A-D). Right ICA angiogram (A) AP view shows direct CCF with anterior and posteroinferior venous drainage. Radiograph of skull (B) AP view shows detachable balloon in the cavernous sinus (white arrow) with coil mass of PAO in the right para sellar region (dashed white arrow). Left ICA angiogram postembolization (C) AP view and right CCA angiogram (D) lateral view show a complete cessation of the shunt. Angiogram and intervention during the recurrence (E, F). Right CCA angiogram (E) lateral view shows recurrence of the left CCF (dashed black arrow) with recanalization of the ICA. Right ECA angiogram (F) AP view shows distal IMA branches contributing to the fistula (black arrow). Radiograph of the skull (G) lateral view shows coil mass in the right parasellar region from transvenous embolization of the right cavernous sinus. Right CCA angiogram $(\mathbf{H})$ shows a complete cessation of the shunt and no opacification of the right ICA.

after coil embolization due to less coil packing density ${ }^{6}$ and after covered stent placement due to endoleak caused by a mismatch in diameter and length between the graft and the fistula. ${ }^{7}$

Other rare causes of recurrence of CCF after successful treatment include the development of indirect CCF and recanalization of the occluded parent artery through vasa vasorum. Indirect CCF has been reported following parent artery occlusion, balloon embolization, and covered stent placement for direct CCF ( - Table 1). The authors have attributed this phenomenon to the angiogenesis triggered by angiogenic factors such as vascular endothelial growth factor (VEGF), which are expressed due to venous hypertension in a high flow CCF or during healing of lacerated dura/CS wall. ${ }^{4,8-10}$ This hypothesis is supported by the results from animal studies, which showed increased VEGF expression promoted by hypoperfusion, sinus hypertension, and sinus thrombosis, leading to the development of dural arteriovenous fistula in rat models. ${ }^{8}$

There are only three reports of recanalization of occluded ICA through vasa vasorum following parent artery occlusion in the literature ( $\boldsymbol{- T a b l e ~} \mathbf{2}$ ), in which all the cases were related to the PAO in giant cerebral aneurysms. ${ }^{11-13}$ Vasa vasorum are small vessels located in the wall of the arteries responsible for microcirculation and providing nutrition. It is postulated that the development of vasa vasorum is induced or influenced by the ischemia, nutritional or metabolic demand of the vessel wall, and/or the organ supplied by the occluded artery..$^{14}$ Single-photon emission computed tomography (SPECT) study had indeed shown that there are reduced cerebral blood flow and altered vasodilatory capacity in the ipsilateral brain, which could act as trigger to the development of vasa vasorum. ${ }^{11}$

We present two cases of recurrence following the endovascular treatment of traumatic CCF. In both the cases, the fistula recurred through the development of indirect CCF. In addition to the dural feeders, the reconstitution of the occluded artery was also observed through the development of vasa vasorum or simple recanalization of the parent artery. The cavernous venous sac was patent in both the cases: in the first patient, the sac was not embolized, and in the second patient, there was deflation of the detachable balloon deployed in the CS. In high-flow direct CCF, the dural arteries may contribute to the CCF that may not be recognized in the initial angiography. These latent fistulae become apparent when the ICA is occluded without the embolization of the CS sac. The cavernous sac then acts as a persistent sump, keeping the fistula patent following the PAO. However, a mere persistence of the dural shunts following PAO cannot explain the observations in our cases. In our case, the indirect CCF was in the proliferative phase, suggesting that dural neovascularization might have been induced by the inflammatory or angiogenetic factors. ${ }^{4,8-10}$ These factors would have played a role in the development of vasa vasorum, leading to the recanalization of the occluded artery. Ischemia of the arterial 

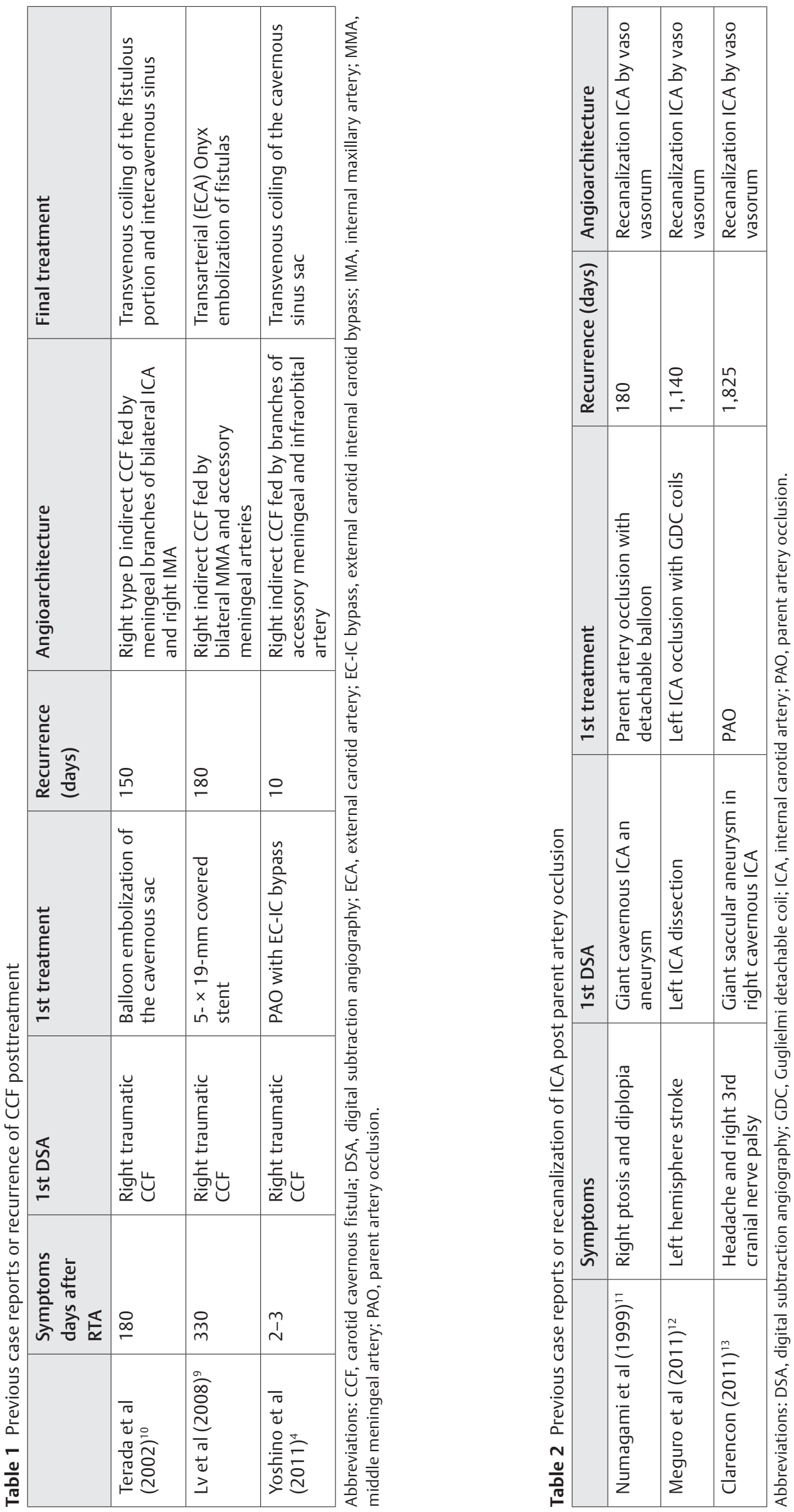
wall secondary to the steal phenomenon due to persistent shunt flow also would have triggered the development of vasa vasorum. ${ }^{14}$

Our observations suggest that the cavernous sac embolization may have to be considered in addition to PAO when PAO is planned as a therapeutic option for direct CCF. Coiling of the sac encourages thrombus formation within the cavernous sac that would eventually lead to complete obliteration when slow or persistent residual flow is present from ICA or dural ECA branches.

\section{Contributions of Authors}

-SK: Data collection, data analysis, data interpretation, manuscript preparation, critical revision

-VM: Manuscript preparation, critical revision

- JER: Manuscript preparation, critical revision

- SKK: Concept and design, data analysis, data interpretation, manuscript preparation, critical revision

\section{Conflict of Interest}

The authors have no personal or financial conflict of interest to disclose.

\section{References}

1 Korkmazer B, Kocak B, Tureci E, Islak C, Kocer N, Kizilkilic O. Endovascular treatment of carotid cavernous sinus fistula: a systematic review. World J Radiol 2013;5(04):143-155

2 Nadarajah M, Power M, Barry B, Wenderoth J. Treatment of a traumatic carotid-cavernous fistula by the sole use of a flow diverting stent. J Neurointerv Surg 2012;4(03):e1

3 Lu X, Hussain M, Ni L, et al. A comparison of different transarterial embolization techniques for direct carotid cavernous fistulas: a single center experience in 32 patients. J Vasc Interv Neurol 2014;7(05):35-47
4 Yoshino H, Ishihara H, Oka F, Kato S, Suzuki M. Development of indirect cavernous dural arteriovenous fistula after trapping for direct carotid cavernous fistula. A case report. Interv Neuroradiol 2011;17(01):104-107

5 Wu Z, Zhang Y, Wang C, Yang X, Li Y. Treatment of traumatic carotid-cavernous fistula. Interv Neuroradiol 2000;6(04):277-289

6 Luo CB, Teng MMH, Chang FC, Lin CJ, Guo WY, Chang CY. Transarterial detachable coil embolization of direct carotidcavernous fistula: immediate and long-term outcomes. J Chin Med Assoc 2013;76(01):31-36

7 Li J, Lan ZG, Xie XD, You C, He M. Traumatic carotid-cavernous fistulas treated with covered stents: experience of 12 cases. World Neurosurg 2010;73(05):514-519

8 Chen L, Mao Y, Zhou LF. Local chronic hypoperfusion secondary to sinus high pressure seems to be mainly responsible for the formation of intracranial dural arteriovenous fistula. Neurosurgery 2009;64(05):973-983, discussion 983

9 Lv X-L, Li Y-X, Liu A-H, et al. A complex cavernous sinus dural arteriovenous fistula secondary to covered stent placement for a traumatic carotid artery-cavernous sinus fistula: case report. J Neurosurg 2008;108(03):588-590

10 Terada T, Miyatake N, Naka D, et al. Indirect carotid cavernous fistula appeared after balloon embolization of direct CCF. Acta Neurochir (Wien) 2002;144(05):489-492

11 Numagami Y, Ezura M, Takahashi A, Yoshimoto T. Antegrade recanalization of completely embolized internal carotid artery after treatment of a giant intracavernous aneurysm: a case report. Surg Neurol 1999;52(06):611-616

12 Meguro T, Muraoka K, Terada K, Hirotsune N, Nishino S. Recanalisation of the internal carotid artery via the vasa vasorum after coil occlusion. Br J Radiol 2011;84(998):e23-e26

13 Clarençon F, Bonneville F, Boch AL, Lejean L, Biondi A. Parent artery occlusion is not obsolete in giant aneurysms of the ICA. Experience with very-long-term follow-up. Neuroradiology 2011;53(12):973-982

14 Cho HJ, Roh HG, Chun YI, Moon CT, Chung HW, Kim HY. Hypertrophy of the vasa vasorum: vascular response to the hungry brain. Neurologist 2012;18(03):133-135 